\title{
El Desarrollo de la Inferencia Analógica en Niños que Viven en Sectores Urbanos Pobres
}

\author{
The Development of Analogical Inference in Children Living in Slums
}

\author{
Mariela Orozco-Hormaza ${ }^{*}, a$ \& Elda Cerchiaro-Ceballos ${ }^{b}$ \\ ${ }^{a}$ Universidad Del Valle, Cali, Colombia \& ${ }^{b}$ Universidad del Magdalena, Santa Marta, Colombia
}

\begin{abstract}
Resumen
Se describe el desarrollo de la inferencia analógica en 405 niños de 3, 4 y 5 años, que viven en sectores urbanos pobres de las ciudades Colombianas de Cali y Santa Marta. Con este fin, se les propuso individualmente un problema de inferencia analógica. Los resultados muestran que niños de todas las edades son capaces de solucionar el problema utilizando la estrategia que requiere establecer inferencia y relaciones de analogía, aunque el porcentaje de niños de 5 años que la usa es significativamente mayor que el de los otros dos grupos. Los resultados obtenidos permiten discutir dos tendencias en el desarrollo cognitivo: una centrada en la edad y la otra en el variabilidad intra e inter-individual.

Palabras clave: Desarrollo cognitivo, inferencia, inferencia analógica, pobreza.

Abstract

The development of analogical inference is described through the study of 405 children aged 3, 4 and 5 years old, living in poor urban neighborhoods in two Colombian cities - Cali (204) and Santa Marta (201). In order to accomplish the objective, children were asked to individually solve an inference problem based on analogical relations. Independently of their age, children were able to solve the problem using a strategy that requires establishing inference and analogical relations. Nevertheless, a higher percentage of the 5-year-old children tend to use that strategy more often. The results obtained allow the discussion of two conceptions of development: one focused on the age and the other on intra- and interindividual variability.

Keywords: Cognitive development, inference, analogical inference, poverty.
\end{abstract}

En el campo del desarrollo cognitivo el concepto de inferencia analógica resulta diverso en función de: (a) la manera como se conceptualiza el proceso, (b) cuándo se desarrolla y (c) la metodología que se utiliza para estudiarla.

Para Piaget, Montangero y Billeter (1977), la inferencia analógica (correlatos, según el traductor) consiste en "desenvolver relaciones entre relaciones basadas en las propiedades intrínsecas de los objetos comparados" (p. 95). DeLoache, Miller y Pierroutsakos (1998) trabajando el razonamiento informal señalan que "el razonamiento inferencial se apoya en la representación mental de relaciones entre entidades" (p. 826). El uso de la repre- sentación "de la relación entre x y y" permite derivar una inferencia "de lo que se conoce de x a y"; esto es, algo "que se conoce de $\mathrm{x}$ y la relación entre $\mathrm{x}$ y y permiten concluir algo sobre y" (DeLoache et al., 1998, p. 805).

DeLoache et al. (1998) igualmente proponen que para inferir los niños utilizan cinco tipos de "relaciones representacionales": de similitud perceptual, conceptual, analógica, simbólica y de reglas. Las relaciones analógicas se basan en la similitud relacional e involucran la aplicación de las relaciones conocidas entre un grupo de elementos a otro grupo diferente.

Si sabemos que a y b están relacionadas de alguna manera y si adicionalmente sabemos que $\mathrm{x}$ tiene la misma relación con a que la que tiene con $b$, podemos asumir que $\mathrm{x}$ y y están relacionados de la misma manera que a y b lo están. (DeLoache et al., 1998, p. 805).

Por supuesto esta diversidad de concepciones incide en el debate que se establece en relación con el desarrollo de la inferencia analógica. Al respecto se discuten dos tesis opuestas y una intermedia: (a) inicialmente se planteó que el desarrollo de la inferencia analógica está ligado con el período de las operaciones formales, (b) posteriormente, algunos autores identificaron la capacidad de 
los bebés para establecer inferencias y analogías, (c) un tercer grupo, propuso una progresión en el desarrollo de la inferencia analógica en función de la edad y del tipo de conocimiento que la fundamenta.

En el primer grupo se pueden ubicar los estudios de Piaget et al. (1977), los post-piagetianos y los teóricos del procesamiento de la información(Ver Goswami, 1991 para un resumen de los estudios realizados por los dos últimos grupos), quienes trabajan el desarrollo de las relaciones analógicas y señalan que solamente en el periodo de las operaciones formales los niños son capaces de hacer inferencias analógicas. Esta condición se deriva de estudios que manejan tareas y criterios que poseen las siguientes características: presentan a los niños tareas de analogía clásica, de tipo verbal, que les exigen establecer relaciones de alto orden entre los cuatro elementos que las componen a:b y c:d, y saber decir y argumentar, generando demandas que dificultan su solución a los más pequeños. Piaget et al. (1977) inician este tipo de estudios utilizando la tarea clásica de analogía. En sesiones individuales, con niños entre 5 y 12 años, entregan un conjunto no ordenado de dibujos - un perro, un pájaro, un barco, una bicicleta etc. y una pluma, un timón de barco, un manubrio, etc. - que en principio deben describir, posteriormente aparear con el elemento apropiado (pájaro: plumas) y finalmente conformar una cuaterna. Si el niño no forma las parejas, el entrevistador pregunta: ¿Qué le ayuda al pájaro a mantenerse caliente? Y luego: ¿Cuál dibujo iría bien aquí (señalando el lugar donde completa la cuaterna). Si el niño no logra establecer la relación, le presentan tres láminas entre las cuales puede escoger aquella que la completa. Para verificar la estabilidad de la relación que establece, el niño debe explicar la elección del elemento y argumentar el contra-argumento que los investigadores le formulan. Estos constituyen los criterios que permiten a Piaget y sus colaboradores aceptar que los niños establecen una analogía y con base en ellos concluyen que sólo en la etapa de las operaciones formales son capaces de utilizarla.

Algunos autores plantean que los bebés son capaces de hacer inferencias analógicas; sin embargo, resulta necesario señalar que ellos utilizan métodos y criterios totalmente diferentes a los de Piaget y sus colaboradores.

Los estudios de Usha Goswami y colaboradores con niños pre-escolares se ubican en la perspectiva del desarrollo temprano del razonamiento analógico y trabajan con tareas que exigen a los niños saber hacer pues no demandan respuestas verbales sino escoger la tarjeta correcta. Goswami y Brown (1989) proponen a niños entre 3 y 6 años versiones de la tarea clásica de analogía $a: b:: c: d$, basada en relaciones entre objetos familiares, como manzanas, chocolate, etc., sometidos a cambios "causales", que los transforman, como derretir y cortar: plastilina:plastilina derretida::manzana:manzana cortada. Los niños nombran los dibujos que las investigadoras organizan en secuencia y completan el elemento d en la secuencia a:b::c:?, seleccionándolo entre cinco tarjetas disponibles. Encuentran que aún los niños de 3 años razonan por analogías y concluyen que el éxito al resolver la tarea está significativamente relacionado con el conocimiento de la relación causal que le sirve de base.

Frente a la discusión sobre el desarrollo temprano o tardío del razonamiento analógico, Halford (1993) adopta una posición intermedia y propone una "progresión en el desarrollo muy relacionada con las edades que corresponden con los estadios piagetianos" (p. 816) y con el tipo de información que se proporciona a los niños. Señala que inicialmente son capaces de establecer relaciones de primer orden basadas en la similitud de los objetos y posteriormente, las de segundo orden, fundadas en la similitud relacional. Gentner (1989, citado por DeLoache et al., 1998) igualmente plantea que los pequeños reconocen las similitudes superficiales o perceptuales y que a medida que la edad y el conocimiento aumentan, se vuelven más sensibles a la estructura relacional subyacente. Finalmente, Richland, Morrison y Holyoak (2006) sugieren que los cambios en el razonamiento analógico a través de las edades dependen de la interacción entre el aumento del conocimiento sobre la relación con la capacidad para integrar múltiples relaciones y el control inhibitorio de las características de la distracción.

El panorama descrito permite concluir que existen diferencias en la conceptualización de la inferencia analógica y en la metodología utilizada para su estudio y que el periodo o la edad en la que los niños son capaces de establecer inferencias y analogías depende tanto de la manera como los investigadores estructuran las tareas como de las exigencias que éstas les crean - saber decir o saber hacer - así como de los criterios que permiten aceptar que un niño es capaz de establecer una inferencia analógica.

Desde la perspectiva de la estructura de las tareas, los psicólogos han investigado el razonamiento analógico utilizando dos tipos de tareas: las pruebas clásicas y las de resolución de problemas (Goswami, 1991). Collins y Burstein (1989) diferencian entre la analogía clásica y los problemas de analogía, en función del número de elementos a manipular y de la estructura de las tareas. En el caso de la analogía clásica los niños deben manipular cuatro elementos a:b::c:d; y establecer comparaciones entre los componentes de cada pareja y entre las parejas. En tanto que los problemas de analogía solamente exigen la comparación entre dos elementos: un elemento original para el que se busca otro comparable.

En relación con la estructura, Holyoak (1984, citado por Goswami, 1991) señala que las dos tareas son funcionalmente diferentes: la analogía clásica "requiere del pensamiento analógico y su objetivo es resolver la analogía, mientras que en el problema analógico, el objetivo es resolver el problema y la habilidad consiste en darse cuenta que se requiere utilizar la analogía" (p. 3). Sin embargo 
Goswami (1991) plantea que desde la perspectiva del desarrollo, "los problemas analógicos no parecen ser más simples que las analogías clásicas” (p. 18).

A manera de resumen, entendemos por inferencia analógica el funcionamiento cognitivo que permite relacionar objetos o entidades, basándose en las propiedades comunes existentes entre ellos. Para estudiar su desarrollo utilizaremos un problema de analogía porque creemos que la resolución de problemas constituye una mejor vía para aproximarnos al funcionamiento cognitivo de niños que viven en entornos urbanos pobres, pues la solución del problema se centra en la actividad del niño y de esta manera permite describir sus estrategias y disminuir el sesgo de información que las tareas verbales pueden generar.

\section{Método}

\section{Diseño}

Se utilizó un diseño transversal, con un grupo de 405 niños, que habitan barriadas pobres de dos ciudades colombianas: Cali (204) y Santa Marta (201), diferenciados en función de su edad, quienes resolvieron un problema de inferencia analógica.

\section{Sujetos}

Participaron en el estudio 134 niños y niñas de 3 años, (edad promedio 41 meses, $S D$ 3.82), 136, de 4 años (edad promedio 53 meses, $S D 3.41$ ) y 135, de 5 años (edad promedio 64 meses, $S D$ 3.66). Los niños asisten a 96 Hogares Comunitarios en Cali (40) y en Santa Marta (56), que atienden a niños cuyas familias viven en barrios pobres. La selección de los niños se hizo atendiendo al criterio edad y ubicación del Hogar Comunitario.

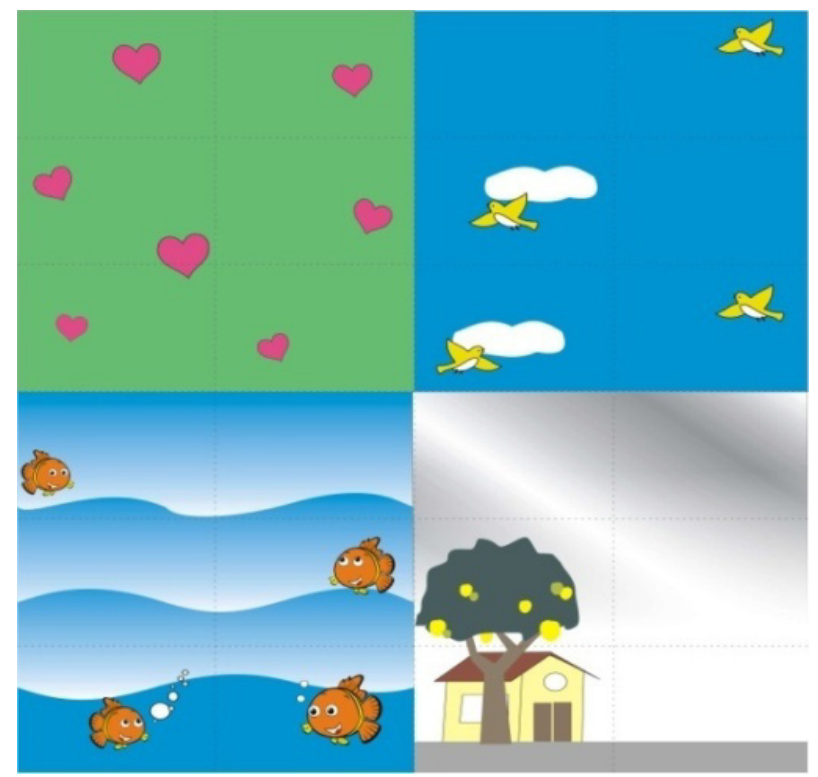

Figura 1A. Tablero descubierto.

\section{El Problema de Inferencia y su Análisis}

Como problema de inferencia analógica se utilizó la tarea de "Las chicas súper poderosas", diseñada por Puche (2003), cuyo objetivo es buscar a tres chicas ocultas en un tablero cubierto por tarjetas removibles. Los materiales de la tarea son: (a) Las siluetas de las tres chicas súper-poderosas; los vestidos de las siluetas y los movimientos que el entrevistador hace corresponden con la actividad que cada chica realiza: nadar, caminar y volar. (b) Un tablero dividido en cuatro cuadrantes, tres de ellos con dibujos que delimitan los entornos en los cuales cada chica realiza su actividad y un cuadrante neutro, que sólo contiene dibujos de corazones; solamente en dos cuadrantes se presentan figuras de animales cuya actividad es similar a la que las chicas realizan. (Ver Figura 1A. Tablero descubierto). (c) Veinticuatro tarjetas para cubrir el tablero y ocultar los dibujos de las chicas. Las tarjetas contienen los mismos dibujos que aparecen en el tablero, menos los de las tres chicas. (Ver Figura 1B. Tablero cubierto).

El análisis de la tarea permite identificar sus elementos constitutivos, a saber:

1. Las actividades asignadas a las siluetas de las tres chicas: $\mathrm{a}=$ voladora, $\mathrm{a}$ " = nadadora, $\mathrm{a} "=$ caminante.

2. Dos tipos de indicios de actividad: dibujos de animales cuyas actividades corresponden con el tipo de actividad de las chicas: pájaros volando (b), peces nadando (b'); y dibujos de los entornos que posibilitan cada tipo de actividad: nubes $\left(b_{1}\right)$, olas o corriente de agua $\left(b_{1}{ }^{\prime}\right)$ y una casa y una calle $\left(b_{1}{ }^{\prime}\right)$.

3. La ubicación de cada indicio de actividad que corresponde con la ubicación de cada chica buscada: la ubicación de pájaros o nubes (c); peces, olas o corriente (c') y casa y calle (c").

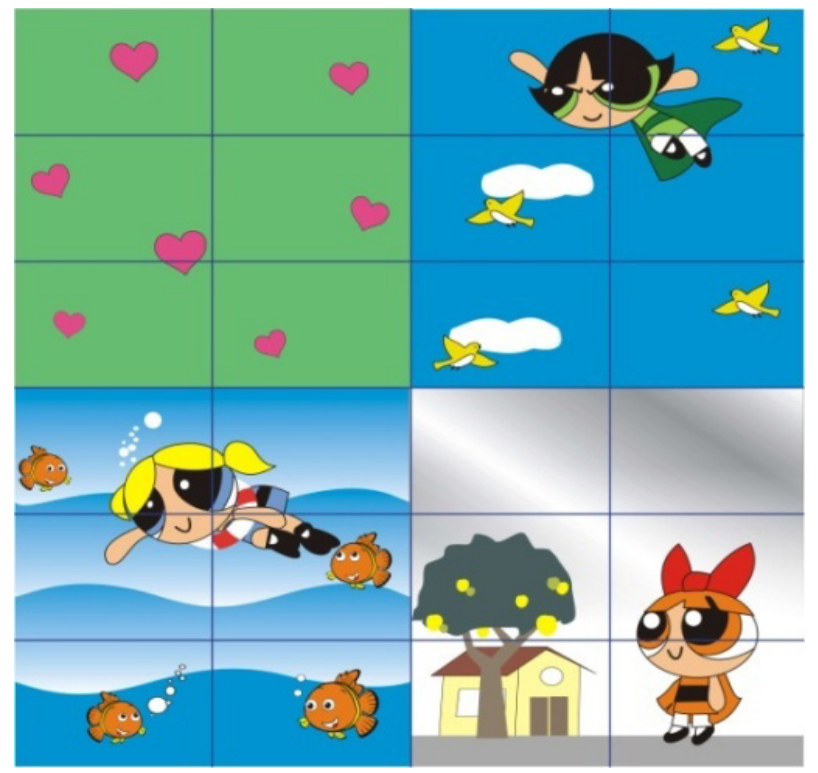

Figura 1B. Tablero cubierto. 
4. Las chicas ocultas: chica voladora (d); chica nadadora (d') y chica caminante (d").

Este problema de analogía presenta al niño dos términos: las chicas y los animales o los entornos y un objetivo 'target': encontrar donde se oculta cada una de las tres chicas. Para resolver este problema, el niño debe generar un ejecutivo (Pascual-Leone \& Johnson, 1991) que lo lleve a buscar a cada chica en el correspondiente cuadrante. La búsqueda exige que el niño:

1. Infiera relación de semejanza a:b, es decir, relacione las actividades asignadas a las siluetas de las chicas (a, a', a") con las actividades de los animales $\left(b, b\right.$ ') o con los entornos que las posibilitan $\left(b_{1}\right.$, $\left.b_{1}, b_{1}{ }^{\prime \prime}\right)$.

2. Infiera relación de semejanza a:c, esto es, relacione las actividades asignadas a las siluetas de las chicas (a, a', a") y las ubicaciones de los indicios de actividad (c, c', c").

3. Establezca relación de identidad c:d, o sea, relacione las ubicaciones de los indicios de actividad (c, c', c") y la ubicación de la chica buscada (d, d', d").

4. Establezca relación de semejanza perceptiva entre chica escogida y chica encontrada.

El análisis de cada elemento constitutivo de la tarea revela que los niños deben relacionar la actividad de las chicas - que corresponde con las características de su atuendo y con los movimientos que el entrevistador realiza - con las actividades de los animales o de los entornos. Esto implica que las propiedades que los niños deben identificar, constituyen "propiedades intrínsecas de los objetos comparados." (Piaget et al., 1977, p. 95) y por lo tanto son relaciones de segundo orden (Gentner, 1988, 1989, citado por DeLoache et al., 1998; Piaget et al., 1977) pues relacionan características no obvias de la pareja a:b. Los dos tipos de relaciones que los niños deben establecer entre chicas y animales o entornos están claramente diferenciadas: de semejanza de las actividades y de semejanza en la ubicación. El hecho de relacionar "las actividades de las chicas con las actividades de los animales" o "las actividades de las chicas con los entornos" no les permite definir la ubicación de la chica.

La relación de semejanza entre las características o propiedades de los objetos no permite resolver el problema pues el niño debe buscar y encontrar el término d, que resulta perceptualmente similar al término a, pero que se encuentra escondido en una ubicación relacionada con los indicios de actividad; por lo tanto, lo que el niño debe identificar es la ubicación del objeto oculto. Por esto consideramos que los niños deben aplicar la semejanza entre las propiedades para definir la ubicación del término d; esto es, establecer una relación probablemente cercana a la analogía. Y decimos que probablemente cercana, porque algunos criterios para las analogías propuestos por Goswami (1991) y Piaget et al. (1977) en unos casos se cumplen y en otros no. Según estos autores, los objetos puestos en relación deben ser claramente diferenciados y esta condición no se cumple pues los objetos del segundo par de la analogía son idénticos a los términos del primer par. Por esto consideramos que se trata de un problema de analogía cuyo objetivo es resolver el problema y para esto se requiere utilizar un cierto tipo de analogía (Holyoak, 1984, citado por Goswami, 1991).

\section{Los Procedimientos de Aplicación y Registro}

La aplicación de la tarea fue precedida de una fase de familiarización. En una y otra se presenta al niño el tablero cubierto por las tarjetas (Figura 1B). En la fase de familiarización el entrevistador se asegura que el niño identifique la actividad que cada chica realiza y si no lo hace, le muestra y dice la actividad de cada chica. Igualmente se asegura que el niño identifique todos los dibujos en las tarjetas que cubren el tablero. En la aplicación propiamente dicha, el entrevistador dice:

En este tablero están escondidas las tres chicas 'super-poderosas': la que está caminando, la que está volando y la que está nadando. Tú vas a descubrir en qué lugar del tablero está cada una de ellas. ¿Cuál de las tres chicas vas a buscar primero? [el entrevistador coloca las siluetas de la tres chicas sobre la mesa]

Cuando el niño elige una chica, le dice: “¿En qué lugar del tablero vas a buscar a esta chica que está...? (nadando, caminando o volando, según la elección del niño)". La tarea se aplicó individualmente, cada sesión fue filmada. Posteriormente se registró la actividad de cada niño en una rejilla que permitió anotar sus verbalizaciones y acciones al resolverla, el orden de búsqueda y la secuencia de acciones al levantar o señalar las tarjetas en el tablero.

\section{El Análisis de los Registros}

Para describir el desarrollo cognitivo del niño se proponen dos niveles de análisis: el primero define su desempeño y se adopta como criterio si encuentra (1) o no encuentra (0) la chica. En el segundo nivel se identifican las estrategias que los niños utilizan. Para esto se tiene en cuenta si manejan o no los indicadores del proceso de solución, al igual que los esquemas de acción involucrados en las estrategias (Ver Tabla 1).

A pesar de sus diferencias, denominamos las tres primeras estrategias como "búsqueda al azar" (Ver Tabla 1) y las dos siguientes como estrategias de evidencia e inferencia. Las tres primeras estrategias no permiten encontrar las chicas. El ejecutivo de búsqueda lleva al niño a utilizar los siguientes procedimientos: levanta tarjetas en cualquier cuadrante, sin reconocer la chica que busca cuando la encuentra (E1); levanta tarjetas en un cuadrante que no corresponde con el de la chica buscada (E2); levanta dos o más tarjetas con indicios en el cuadrante de la chica buscada para luego levantar tarjetas en cualquier otro cuadrante (E3); por esto las denominamos de azar. En la estrategia de evidencia (E4), levanta tarjetas al azar y encuentra una parte de la chica que busca, reconoce la 
Tabla 1

Descripción de Estrategias de Búsqueda

\begin{tabular}{|c|c|c|}
\hline Puntaje & Estrategia de búsqueda & Esquemas de Acción \\
\hline 1 & E1. Búsqueda al azar & $\begin{array}{l}\text { 1. Activa esquema de búsqueda, levantando diferentes } \\
\text { tarjetas. }\end{array}$ \\
\hline 2 & $\begin{array}{l}\text { E2. Búsqueda al azar limitada } \\
\text { a un cuadrante }\end{array}$ & $\begin{array}{l}\text { 1. Activa esquema de búsqueda } \\
\text { 2. Genera organización espacial del tablero o define } \\
\text { un espacio de búsqueda }\end{array}$ \\
\hline 3 & $\begin{array}{l}\text { E3. Búsqueda al azar } \\
\text { y reconocimiento de indicios }\end{array}$ & $\begin{array}{l}\text { 1. Activa esquema de búsqueda. } \\
\text { 2. Establece relación de semejanza entre la actividad asignada } \\
\text { a una chica (a, o a', o a") y la actividad del indicio (b, b') }\end{array}$ \\
\hline 4 & $\begin{array}{l}\text { E4. Búsqueda al azar } \\
\text { y reconocimiento de evidencia }\end{array}$ & $\begin{array}{l}\text { 1. Activa esquema de búsqueda. } \\
\text { 2. Establece relación de parte a todo o "pertenencia partitiva" } \\
\text { 3. Establece relación de semejanza perceptiva entre chica } \\
\text { escogida y chica encontrada. }\end{array}$ \\
\hline 5 & $\begin{array}{l}\text { E5. Búsqueda por indicios } \\
\text { y reconocimiento de evidencia }\end{array}$ & $\begin{array}{l}\text { 1. Activa esquema de búsqueda. } \\
\text { 2. Infiere relación de semejanza entre las actividades } \\
\text { asignadas a las siluetas de las chicas }(\mathrm{a}, \mathrm{a}, \mathrm{a}, \mathrm{a}) \text { y las } \\
\left.\text { actividades de los indicios }(\mathrm{b}, \mathrm{b}, \mathrm{b},), \mathrm{o}\left(\mathrm{b}_{1}, \mathrm{~b}_{1}\right)^{\prime}\right) \\
\text { 3. Infiere relación de semejanza entre las actividades } \\
\text { asignadas a las siluetas de las chicas (a, a', a') } \\
\text { y las ubicaciones de los indicios de actividad (c, c', c"). } \\
\text { 4. Establece relación de semejanza entre las ubicaciones } \\
\text { de los indicios de actividad (c, c', c') y la ubicación } \\
\text { de la chica buscada (d, d', d'). } \\
\text { 5. Establece relación de semejanza perceptiva entre chica } \\
\text { escogida y chica encontrada. }\end{array}$ \\
\hline
\end{tabular}

evidencia y continúa levantando las tarjetas contiguas a la evidencia encontrada. Estos niños establecen relación de parte a todo o "pertenencia partitiva" (Piaget \& Inhelder, 1976, p. 63) entre cualquier parte de la chica buscada y el resto de su cuerpo. Solamente la estrategia 5 (E5) exige establecer relación entre la actividad de la chica buscada, la actividad o características de los indicios y la ubicación de los mismos, o sea, inferir las relaciones de semejanza entre $a: b, a: c$ y c:d.

Como las estrategias 1, 2, 3, se caracterizan por búsquedas regidas por el azar y no permiten encontrar las chicas, se les asigna un valor de 1; a las otras dos estrategias (E4 y E5) que permiten encontrarlas, se asigna un valor 4 y 5, respectivamente. Para obtener el puntaje final de la tarea, se identifican las estrategias que cada niño efectivamente utiliza en las tres búsquedas y se promedian los puntajes para las tres búsquedas.

\section{Resultados}

La presentación de los resultados incluye el análisis del desempeño de los niños al resolver la tarea y el de las estrategias que emplean en cada búsqueda.

La Figura 2 presenta la distribución de los porcentajes de niños por grupo de edad de acuerdo con su desempeño. El porcentaje de niños que encuentra las tres chicas aumenta en función de la edad (22\% a los 3 años, $28 \%$ a los 4 y $37 \%$, a los 5). El porcentaje de niños que logran encontrar dos chicas disminuye a medida que la edad aumenta (44\% a los 3 años, $38.2 \%$ a los 4 y $37 \%$ a los 5).

La Tabla 2 muestra la distribución del porcentaje de niños según la estrategia que utilizan en cada búsqueda, en función de la edad. 
Orozco-Hormaza, M. \& Cerchiaro-Ceballos, E. (2012). El Desarrollo de la Inferencia Analógica en Niños que Viven en Sectores Urbanos Pobres.

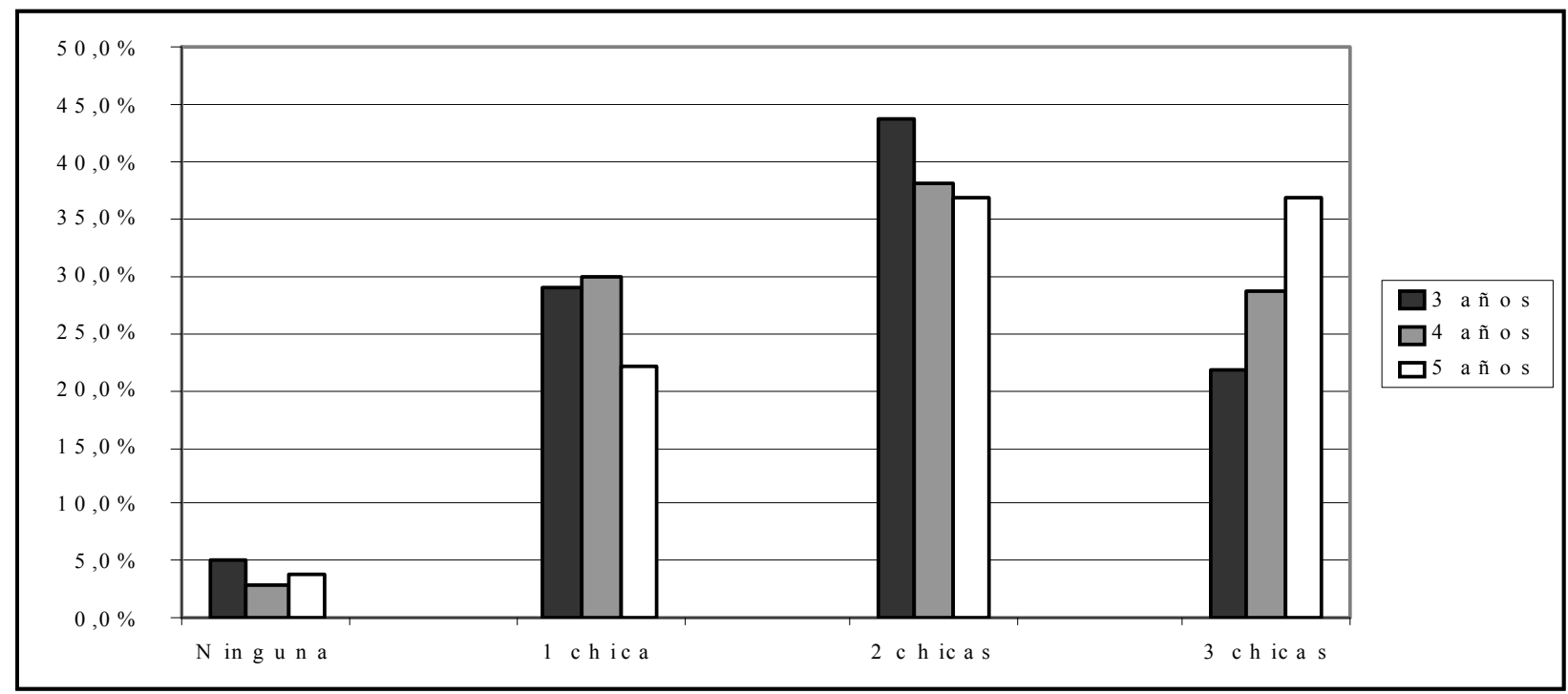

Figura 2. Distribución del porcentaje de niños en función de número de chicas encontradas y edad.

Tabla 2

Distribución del Porcentaje de Niños según Tipo de Estrategia Utilizada en Cada Búsqueda y Edad

\begin{tabular}{|c|c|c|c|c|}
\hline Edad & Tipo de estrategia & Búsqueda $1 \%$ & Búsqueda $2 \%$ & Búsqueda $3 \%$ \\
\hline \multirow[t]{5}{*}{3} & 1. Azar & 19.4 & 20.9 & 15.7 \\
\hline & 2. Azar limitado a un cuadrante & 21.6 & 14.2 & 4.5 \\
\hline & 3. Azar y reconocimiento de indicios & 16.4 & 9.7 & 0.7 \\
\hline & 4. Azar y reconocimiento de evidencia & 17.2 & 38.1 & 50.0 \\
\hline & 5. Indicios y reconocimiento de evidencia & 25.4 & 17.2 & 29.1 \\
\hline \multirow[t]{5}{*}{4} & 1. Azar & 21.3 & 18.4 & 13.2 \\
\hline & 2. Azar limitado a un cuadrante & 19.9 & 15.4 & 2.9 \\
\hline & 3. Azar y reconocimiento de indicios & 8.8 & 9.6 & 0.7 \\
\hline & 4. Azar y reconocimiento de evidencia & 24.3 & 30.1 & 50.7 \\
\hline & 5. Indicios y reconocimiento de evidencia & 25.7 & 26.5 & 32.4 \\
\hline \multirow[t]{5}{*}{5} & 1. Azar & 20.7 & 20.7 & 11.1 \\
\hline & 2. Azar limitado a un cuadrante & 16.3 & 9.6 & 2.2 \\
\hline & 3. Azar y reconocimiento de indicios & 5.9 & 5.9 & 0.7 \\
\hline & 4. Azar y reconocimiento de evidencia & 23.0 & 25.9 & 31.1 \\
\hline & 5. Indicios y reconocimiento de evidencia & 34.1 & 37.8 & 54.8 \\
\hline
\end{tabular}

La suma del porcentaje de niños que utilizan las estrategias 4 y 5 , en función de la edad, muestra un incremento en todas las edades, a través de las tres búsquedas. A los 3 años, $42.6 \%$ las utilizan en la búsqueda de la primera chica; el $55.3 \%$, en la segunda y el $79.1 \%$, en la tercera. A los 4 años, el 50.0\% las utilizan en la primera; $56.6 \%$, en la segunda y el $83.1 \%$, en la tercera. A los 5 años, $57.1 \%$ las utilizan en la primera, $63.7 \%$, en la segunda y $85.9 \%$, en la tercera. El mismo análisis aplicado a cada búsqueda muestra que los porcentajes de éxito igualmente difieren en función de la edad.

Un ANOVA de dos vías de clasificación permitió encontrar diferencias significativas en el porcentaje de los niños que usan los dos tipos de estrategias: de evidencia (E4) e inferencia (E5) y las de azar (E1, E2 y E3), en todas las edades, a través de las tres búsquedas. Para los 3 años $F(2,401)=23,78, P<.001$; para 4 años $F(2,407)=$ $18,94, P<.001$; para 5 años $F(2,404)=14,17, P<.001$.

La utilización de un ANOVA de dos vías de clasificación, permitió igualmente encontrar diferencias significativas en los porcentajes de utilización de un tipo de estrategias, en función de la edad, a través de las tres búsquedas. Los niños de 4 años presentan variaciones significativas entre la estrategia de evidencia (E4) y las demás estrategias $F(4,14)=5.89, P<.05$; y los de 5 años, entre la estrategia de inferencia (E5) y las demás estra- 
tegias $F(4,14)=14.92, P<.001$. No se encontró diferencia significativa entre las estrategias que los niños de 3 años utilizan.

La combinatoria de las estrategias utilizadas por cada niño en las tres búsquedas presenta una alta variabilidad: se obtuvieron 21 combinatorias efectivas de las 27 posibles. La Figura 3 muestra la distribución del porcentaje de niños, en función de la combinatoria de las tres estrategias y de la edad. La gráfica revela que niños de todas las edades utilizan tanto las estrategias menos elaboradas como las más elaboradas.

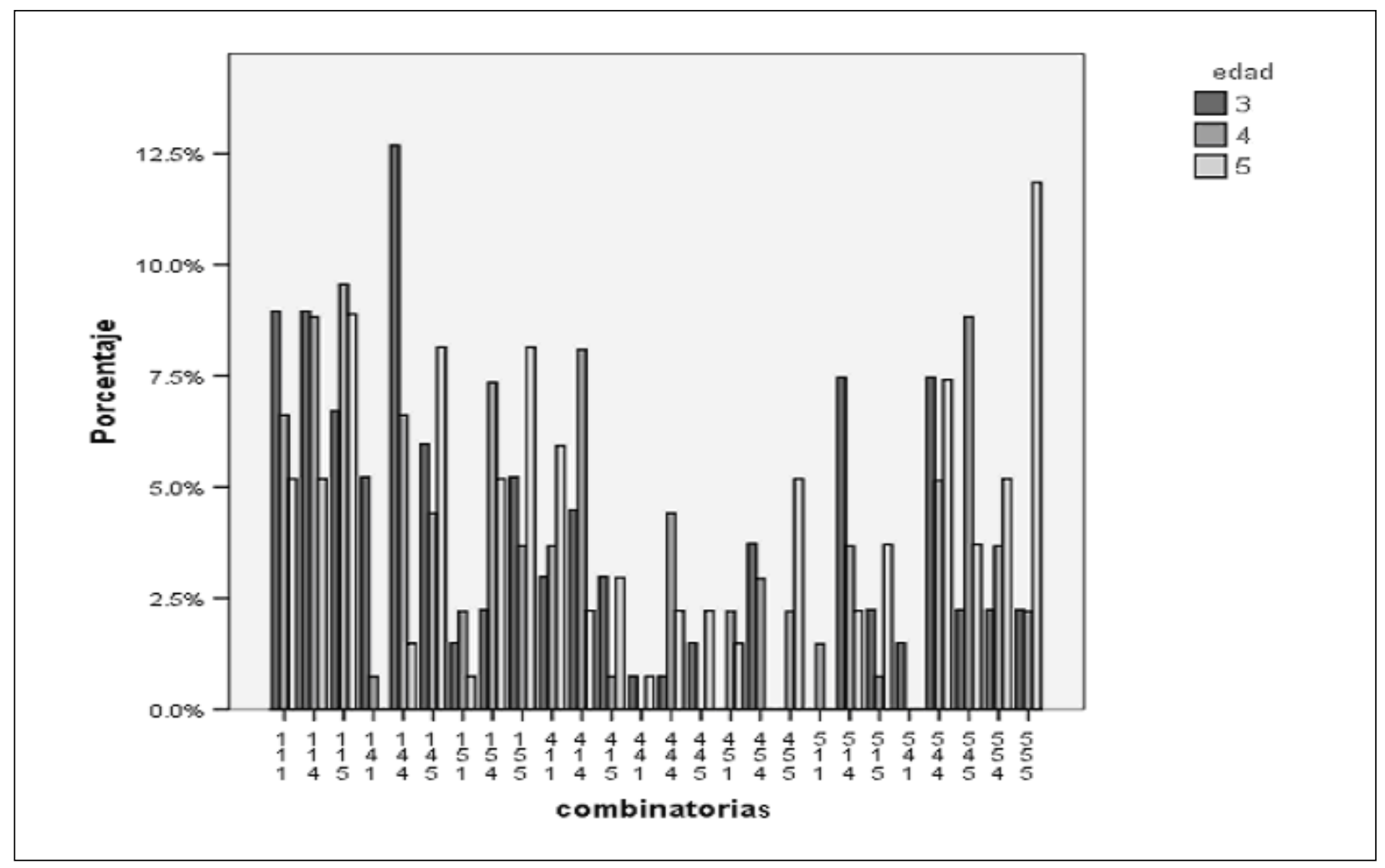

Figura 3. Distribución de porcentaje de niños asignados a combinatoria de estrategias en las tres búsquedas, en función de la edad (el orden de las estrategias en las rutas se lee de abajo hacia arriba).

Las combinatorias de las estrategias permiten definir las "rutas" de búsqueda que los niños siguen (Siegler \& Stern, 1998), es decir, la secuencia de estrategias que utilizan a través de las tres búsquedas.

En relación con las rutas que los niños tienden a seguir se pueden señalar dos tendencias: niños de todas las edades utilizan la ruta 555; sin embargo, el porcentaje de niños de 5 años que la usa (11.9\%) supera el de las otras dos edades $(2.2 \%)$. La ruta más utilizada por los niños de tres años es la ruta ascendente (144) que presenta el mayor porcentaje de casos para esa edad (12.7\%), en tanto que para los de cuatro años, la ruta 115 es la más utilizada (9.6\%).

Se establecieron dos tipos de rutas en función de la variabilidad y el orden de las mismas: estables y variables.

1. Las rutas estables se caracterizan por el uso del mismo tipo de estrategia en las tres búsquedas, son: (111) azar, azar, azar; (444) evidencia, evidencia, evidencia y (555) inferencia, inferencia, inferencia. La ruta 111 no permite a los niños encontrar chica alguna, en cambio, las combinatorias 444 y 555 les permiten en- contrar a todas las chicas. El porcentaje más alto de niños que utilizan estas dos últimas rutas se ubican en el grupo de 4 años, que en un $4.4 \%$ sigue la ruta centrada en la evidencia (444), y $11.9 \%$ de los de 5 años sigue la ruta centrada en la relación de inferencia (555). Los niños de 3 años presentan el porcentaje más alto $(9 \%)$ en la ruta 111 .

2.Las rutas variables, caracterizadas por las fluctuaciones en el tipo de estrategia utilizada, presentan tres tipos de variaciones: (a) Las rutas ascendentes, se caracterizan por el uso progresivo de estrategias que van desde las menos elaboradas hasta las más complejas. En la primera búsqueda, los niños utilizan la estrategia menos elaborada y en la $2^{\circ}$ y/o $3^{a}$, estrategias más elaboradas. Las siguientes combinatorias presentan una ruta ascendente: (144) azar, evidencia, evidencia; (145) azar, evidencia, inferencia; (155) azar, inferencia, inferencia; (445) evidencia, evidencia, inferencia; (455) evidencia, inferencia, inferencia. Los niños de 4 años tienden a utilizar las rutas $114 \mathrm{y}$ 115 (8.8\%, 9.8\% respectivamente). Los niños de 3 
años tienden a usar la ruta $144(12.7 \%)$ y los de 5 , las rutas $145(8.1 \%), 155(8.1 \%)$ y $455(5.2 \%)$. (b) Las rutas descendentes se caracterizan por el uso de estrategias en un orden decreciente de complejidad; inician con estrategias del nivel más alto y terminan con estrategias del nivel más bajo, así: 411, 441, 511, $541,544,554$. Los porcentajes de niños que utilizan las rutas descendentes son bajos, con excepción de la ruta 544, que es seguida por un porcentaje relativamente similar de niños de todas las edades ( 3 años: $7.5 \%$; 4 años: $5.1 \%$ y de 5 años: $7.4 \%$ ). (c) Las rutas inestables muestran una alta fluctuación en el uso de estrategias, pues los niños pasan de una estrategia más o menos elaborada, en la primera búsqueda a otra menos o más elaborada en la segunda y a otra del tipo contrario en la tercera: 141, 151, 154, 414, $415,451,454,514,515,545$. La sumatoria de los porcentajes de niños que en cada grupo de edad utilizan las rutas inestables muestra que los de 4 años tienden a utilizarlas más (36.7\%) que los niños de 3 años $(32 \%)$, en tanto que los niños de 5 años las utilizan en un porcentaje menor (18.5\%).

Tabla 3

Distribución de Porcentajes de Rutas en Función de Edad

\begin{tabular}{|c|c|c|c|}
\hline \multirow{2}{*}{ Tipos de Rutas } & \multicolumn{3}{|c|}{ Edad } \\
\hline & 3 & 4 & 5 \\
\hline 111 & 9.0 & 6.6 & 5.2 \\
\hline 114 & 9.0 & 8.8 & 5.2 \\
\hline 115 & 6.7 & 9.6 & 8.9 \\
\hline 141 & 5.2 & 0.7 & 0.0 \\
\hline 144 & 12.7 & 6.6 & 1.5 \\
\hline 145 & 6.0 & 4.4 & 8.1 \\
\hline 154 & 2.2 & 7.4 & 5.2 \\
\hline 155 & 5.2 & 3.7 & 8.1 \\
\hline 411 & 3.0 & 3.7 & 5.9 \\
\hline 414 & 4.5 & 8.1 & 2.2 \\
\hline 455 & 0,0 & 2.2 & 5.2 \\
\hline 514 & 7.5 & 3.7 & 2.2 \\
\hline 544 & 7.5 & 5.1 & 7.4 \\
\hline 545 & 2.2 & 8.8 & 3.7 \\
\hline 554 & 2.2 & 3.7 & 5.2 \\
\hline 555 & 2.2 & 2.2 & 11.9 \\
\hline
\end{tabular}

\section{Discusión y Conclusiones}

A medida que la edad aumenta, un mayor porcentaje de niños encuentran las tres chicas o sea, los niños de 5 años presentan una ejecución más exitosa que los de 3 y 4 años. Independientemente del grupo de edad, los porcentajes más altos se obtienen cuando los niños encuentran dos chicas.
La utilización del paradigma de resolución de problemas permitió identificar las estrategias de los niños, en las secuencias de acciones que realizan, e inferir su actividad mental. Un porcentaje alto de los niños de 4 y 5 años usan estrategias exitosas de evidencia (E4) y de inferencia (E5) en cada una de las tres búsquedas; inicialmente $50 \%$ y más de los niños de los dos grupos de edad las utilizan y en la tercera búsqueda aumentan hasta el 83 y $86 \%$, respectivamente. Estos porcentajes de acierto para encontrar la chica oculta se pueden homologar con los porcentajes de éxito de niños de todas las edades en los estudios de Goswami y colaboradores (Goswami \& Brown, 1989) al elegir la tarjeta correcta que completa la cuaterna; sin embargo, resulta necesario señalar que en esta investigación la tarea es más difícil pues los niños tienen que descubrir las chicas ocultas y no elegir una tarjeta entre cinco opciones para completar la cuaterna.

Las diferencias significativas encontradas entre el porcentaje de los niños de 4 y 5 años que usan los dos tipos de estrategias exitosas (E4 y E5), a través de las tres búsquedas, permiten señalar que estos niños mejoran significativamente su ejecución durante la resolución del problema pues el uso de estrategias que posibilitan encontrar las chicas se incrementa y el uso de estrategias de azar decrece; sin embargo, solamente los de 5 años presentan diferencias significativas en la utilización de estrategias del más alto nivel relacional. Estos resultados permiten proponer que a medida que resuelven el problema los niños logran centrarse más en el mismo, lo que les permite reorganizar sus esquemas de acción y mejorar sus estrategias y por ende, su desempeño.

Tanto el porcentaje de niños de 5 años que obtienen puntajes de desempeño altos, como el porcentaje de niños de esta edad que infiere semejanzas entre las actividades de las chicas y las de los animales o el correspondiente entorno y establece analogías entre las actividades y su ubicación, son mayores que el porcentaje de niños de 3 y 4 años que logran puntajes altos y utilizan esta estrategia. Sin embargo, tanto los niños de tres años como los de cuatro son capaces de establecer analogías, manejando relaciones de semejanza entre la actividad de dos objetos familiares, claramente diferenciados y su ubicación. Este hecho revela una gran variabilidad en la utilización de estrategias en las tres búsquedas, o sea, en la ruta que cada niño sigue.

Estos resultados permiten ver las dos caras del desarrollo: el desarrollo escalonado en función de la edad y la variabilidad en las ejecuciones en cada niño y en cada grupo de edad. En cada nivel de edad se observa una gran variabilidad en las rutas que siguen a través de las tres búsquedas, variabilidad generada por la variación intraindividual, que se evidencia cuando analizamos las estrategias y no el desempeño.

Actualmente, la psicología del desarrollo cognitivo describiría este fenómeno como un caso típico de variabilidad en la ejecución, que da cuenta del cambio cognitivo (Puche, 2003; Siegler, 2002, 2007). Esta variabilidad se 
puede entender en dos sentidos: resultados diferenciados intra-individuales a través de las tres búsquedas que se expresa en la variabilidad de las rutas y resultados diferenciados inter-individuales, en el mismo grupo de edad.

Es así como al tratar de describir el desarrollo cognitivo de los niños que viven en sectores urbanos pobres nos hemos encontrado las dos caras del desarrollo de la inferencia analógica: la tendencia a un mayor desarrollo a medida que la edad aumenta y las variaciones en cada nivel de edad, producto de la variabilidad intra-individual.

¿Cómo explicar que en una búsqueda el mismo niño use la inferencia analógica y en otra no, o viceversa?. Richland et al. (2006) señalan tres grandes tendencias en los debates sobre desarrollo cognitivo del razonamiento analógico en relación con las diferencias en la edad de aparición: el incremento en el conocimiento con la edad; el cambio en la relación que utilizan: de similitud entre los objetos a una similitud relacional; y el incremento en la capacidad de procesamiento y en la memoria de trabajo para manipular las relaciones. Sin embargo, las tesis de estos autores no permiten explicar por qué, en algunas búsquedas, el conocimiento y la capacidad de procesamiento o la memoria de trabajo sí funcionan y en la siguiente o anterior no. Nuestra hipótesis es que una "descentración" momentánea del niño, puede impedir la activación de los esquemas de búsqueda necesarios para establecer la analogía (Pascual-Leone \& Johnson, 1991); esta tesis podría explicar la variabilidad; sin embargo se necesitaría una investigación de otra índole para probar este supuesto.

Weatherholt, Harris, Burns y Clement (2006) señalan que una atención mental deficiente llevaría a los niños pobres a resultados menos exitosos que los de los niños que pertenecen a un nivel socio-económico medio al resolver tareas de razonamiento analógico. Sin embargo, parece que en este estudio, la atención mental de los niños tiende a aumentar a medida que resuelven los componentes del problema. Se podría entonces concluir, que los niños que viven en entornos urbanos pobres no necesariamente presentan un desarrollo de la inferencia analógica más bajo que los de nivel medio. Posteriores elaboraciones nos permitirán establecer cuales dimensiones en los contextos de interacción familiar estarían relacionadas con el desarrollo bajo de la inferencia analógica, que algunos pocos niños de todas las edades presentan como una constante.

\section{Referencias}

Collins, A., \& Burstein, M. (1989). A framework for a theory of mapping. In S. Vosniadou \& A. Ortony (Eds.), Similarity and analogical reasoning (pp. 546-565). Cambridge, UK: Cambridge University Press.
DeLoache, J., Miller, K., \& Pierroutsakos, S. (1998). Reasoning and problem solving. In W. Damon, D. Khun, \& R. Siegler (Eds.), Handbook of Child Psychology. Cognition, perception and language $\left(5^{\text {th }}\right.$ ed., pp. 801-850). New York: Wiley \& Sons. Gentner, D. (1989). The mechanisms of analogical learning. In S. Vosniadou \& A. Ortony (Eds.), Similarity and analogical reasoning (pp. 199-241). London: Cambridge University Press. (Reprinted in Knowledge acquisition and learning, 1993, 673-694).

Goswami, U. (1991). Analogical reasoning: What develops? A review of research and theory. Child Development, 62(1), 1-22.

Goswami, U., \& Brown, A. (1989). Melting chocolate and melting snowmen: Analogical reasoning and causal relations. Cognition, 35(1), 69-95.

Pascual-Leone, J., \& Johnson, J. (1991). The psychological unit and its role in task analysis: A reinterpretation of object permanence. In M. Candler \& M. Chapman (Eds.), Criteria for competence (pp. 153-187). Hillsdale, NJ: Lawrence Erlbaum.

Piaget, J., \& Inhelder, B. (1976). Génesis de las estructuras lógicas elementales. Buenos Aires, Argentina: Guadalupe.

Piaget, J., Montangero, J., \& Billeter, J. B. (1977). La formación de los correlatos. In J. Piaget, Investigaciones sobre la abstracción reflexionante (pp. 93-104). Buenos Aires, Argentina: Huemul.

Puche, R. (2003). El niño que piensa y vuelve a pensar. Santiago de Cali, Colombia: Artes Gráficas del Valle.

Richland, L. E., Morrison, R. G., \& Holyoak, K. J. (2006). Children's development of analogical reasoning: Insights from scene analogy problems. Journal of Experimental Child Psychology, 94, 249-273.

Siegler, R. S. (2002). Variability and infant development. Infant Behavior \& Development, 25, 550-557.

Siegler, R. S. (2007). Cognitive variability. Developmental Science, 10(1), 104-109.

Siegler, R. S., \& Stern, E. (1998). Conscious and unconscious strategy discoveries: A microgenetic analysis. Journal of Experimental Psychology: General, 127(4), 77-397.

Weatherholt, T., Harris, R., Burns, B., \& Clement, C. (2006). Analysis of attention and analogical reasoning in children of poverty. Applied Developmental Psychology, 27, 125-135. 